\title{
Una perspectiva ontosemiótica de los problemas y métodos de investigación en educación matemática
}

\author{
Juan D. Godino \\ Carmen Batanero \\ María Burgos \\ María M. Gea
}

\begin{abstract}
Resumen: Una crítica tradicional a la investigación científica en el campo educativo ha sido la desconexión con la práctica docente, motivada por el fraccionamiento de los problemas de investigación. Para resolver esta disociación, las investigaciones de diseño instruccional plantean problemáticas de tipo predictivo más próximas a dicha práctica, pero deben ser complementadas con una interfaz que aporte los componentes valorativos y normativos que se deducen de la aplicación eficiente de los resultados de la literatura previa. En este trabajo usaremos algunas herramientas del Enfoque Ontosemiótico (EOS) para esbozar una agenda de investigación que tiene en cuenta los componentes científico (descriptivo, explicativo y predictivo) y tecnológico (valorativo y normativo) de la investigación didáctica. Asimismo, describimos las herramientas metodológicas que aporta el EOS para abordar los mencionados problemas, en particular la noción de idoneidad didáctica que sirve de nexo entre el componente científico-tecnológico y la práctica de la enseñanza.
\end{abstract}

Juan D. Godino

Doctorado en Matemáticas por la Universidad de Granada. Profesor de la

Universidad de Granada, Granada, España.

(D) https://orcid.org/0000-0001-84090258 $\bowtie$ jdgodino@gmail.com

Carmen Batanero Doctorada en Matemáticas por la Universidad de Granada. Profesora de la

Universidad de Granada, Granada, España

Dhttps://orcid.org/0000-0002-4189-

$\triangle$ batanero@ugr.es

María Burgos

Doctorada en Matemáticas por la Universidad de Almería y Doctorada en Didáctica de las Matemáticas por la Universidad de Granada. Profesora de la

Universidad de Granada, Granada, España.

(1) https://orcid.org/0000-0002 4598-7684 $\bowtie$ mariaburgos@ugr.es

María M. Gea Doctorada en Didáctica de las Matemáticas por la Universidad de Granada. Profesora de la Universidad de Granada, Granada, España.

D https://orcid.org/0000-0002$\underline{5229-0121}$ $\bowtie$ mmgea@ugr.es

Recebido em 25/04/2021 Aceito em 07/06/2021 Publicado em 21/06/2021
Palabras clave: Educación matemática. Investigación didáctica. Enfoque Ontosemiótico. Metodología.

\section{Uma perspectiva ontosemiótica dos problemas e métodos de pesquisa em educação matemática}

Resumo: Uma crítica tradicional à pesquisa científica no campo educacional tem sido a desconexão com a prática docente, motivada pela divisão dos problemas de pesquisa. Para resolver essa dissociação, as investigações de design instrucional apresentam problemas preditivos mais próximos a essa prática, mas devem ser complementados com uma interface que forneça os componentes avaliativos e normativos que são deduzidos da aplicação eficiente dos resultados da literatura anterior. Neste trabalho utilizaremos algumas ferramentas da Abordagem Ontosemiótica (OS) para traçar uma agenda de pesquisa que leve em consideração os componentes científicos (descritivos, explicativos e preditivos) e tecnológicos (avaliativos e normativos) da pesquisa didática. Do mesmo modo, descrevemos as ferramentas metodológicas disponibilizadas pela EOS para abordar os problemas referidos, em particular a noção de adequação didática que serve de elo entre a componente científico-tecnológica e a prática docente.

Palavras-chave: Educação Matemática. Investigação didática. Enfoque Ontosemiótico. Metodologia.

\section{An onto-semiotic perspective of the research problems and methods in mathematics education}

Abstract: A traditional criticism of scientific research in the field of education is the disconnection with teaching practice, which is due to the fragmentation of research problems. In order to solve this disconnection, instructional design research poses predictive problems that are closer to such practice, but that should be complemented with an interface providing the evaluative and normative components that are deduced from the efficient application of previous research literature. In this paper we apply some tools from the Ontosemiotic Approach (OSA) to outline a research agenda that takes into account the scientific (descriptive, explanatory and predictive) and 
technological (evaluative and normative) components of didactic research. We also describe the methodological tools provided by OSA to address the aforementioned problems, in particular the notion of didactic suitability that serves as a link between the scientific-technological component and the teaching practice.

Keywords: Mathematics education. Didactic research. Onto-semiotic approach. Methodology.

\section{Introducción}

Como profesores de matemáticas estamos interesados en encontrar respuestas a cuestiones que refieren a la práctica de la enseñanza: ¿Qué contenidos matemáticos deberíamos enseñar a nuestros estudiantes? ¿Cómo tendríamos que enseñarlos de modo que aprendan lo mejor posible? La respuesta a estas cuestiones pasa por la investigación en didáctica de la matemática, que las precisa en términos de problemas abordables según criterios de validez y rigor metodológico propios de la investigación científica y tecnológica. Además, la formulación de estos problemas depende de los marcos teóricos utilizados, los cuales proporcionan principios sobre la naturaleza de la propia matemática, así como sobre su aprendizaje y enseñanza.

Lo que puede resultar menos obvio, pero es igualmente esencial, es que toda investigación empírica está relacionada con supuestos teóricos (a veces tácitos, pero no por ello menos fuertes) que les sirven de base. Incluso las observaciones más sencillas o la recopilación de datos se llevan a cabo bajo el paraguas de premisas teóricas implícitas o explícitas, que dan forma a la interpretación de la información recopilada. Si no se reconoce este hecho y no se actúa adecuadamente sobre él, la investigación puede resultar inútil o engañosa (SCHOENFELD, 2007, p. 70).

El esfuerzo analítico por definir problemas abordables con precisión dentro de la investigación didáctica lleva, en general, a centrar la atención en aspectos puntuales, con una finalidad fundamentalmente descriptiva y explicativa, con frecuencia alejados de la problemática docente del aula. Esta puede ser una de las explicaciones de la brecha entre la investigación y la práctica que, como mencionan diversos autores, es un problema permanente en el campo de la investigación educativa (ENGLISH y KISCHNER, 2016; KORTHAGEN, 2007; MCINTYRE, 2005).

En este trabajo utilizamos algunas nociones del Enfoque Ontosemiótico (EOS) sobre el conocimiento y la instrucción matemáticos (GODINO, 2002; GODINO; BATANERO y FONT, 2007; 2019) para proponer un programa de investigación en educación matemática que tenga en cuenta:

- La especificidad del contenido matemático sobre el que se realiza el diseño de su enseñanza y aprendizaje; 
- La descomposición analítica de los problemas de investigación en sus diferentes facetas implicadas (epistémica, cognitiva, afectiva, interaccional, mediacional y ecológica), atendiendo tanto a la finalidad descriptiva como explicativa de la problemática abordada:

- La identificación de criterios que ayuden, en la práctica docente, al estudio de contenidos matemáticos específicos a propósito de los conocimientos descriptivos y explicativos obtenidos en la investigación.

Comenzamos el trabajo con una descripción breve de algunos antecedentes y nociones del marco teórico EOS que se utilizan posteriormente para formular las cuestiones de investigación. En la Sección 3 presentamos los criterios o dimensiones de clasificación de las cuestiones y las categorías tenidas en cuenta en cada dimensión. En la Sección 4 se describe la Teoría de Idoneidad Didáctica como nexo entre la investigación teórica y la práctica de la enseñanza propuesta por el EOS. En la Sección 5, y sin pretensiones de exhaustividad, incluimos una muestra de enunciados de cuestiones para las diversas dimensiones y categorías propuestas. En la Sección 6 resumimos las principales características del enfoque metodológico que consideramos pertinente para abordar la "agenda de investigación" esbozada en el marco del EOS. Terminamos con algunas reflexiones finales acerca del uso potencial de este trabajo por parte de estudiantes de postgrado e investigadores en el área de Didáctica de la Matemática.

\section{Antecedentes y marco teórico}

El análisis crítico de los problemas de investigación en Educación Matemática ha sido abordado desde varias perspectivas. Una revisión de los manuales de investigación (handbooks) en educación matemática revela las áreas prioritarias sobre las cuales se viene investigando en cada momento, con la finalidad general de mejorar del proceso didáctico. Aun así, en English y Kischner (2016) se resalta la necesidad de adaptar y diseminar los resultados de la investigación de manera que favorezcan el aprendizaje y la enseñanza, reconociendo que existe un vacío entre investigación y práctica cuya solución reclama los puntos siguientes: 1) Repensar los problemas de investigación en educación matemática y las metodologías que se usan para abordarlos; 2) Realizar investigaciones de diseño que incluyan a los profesores como partícipes de la investigación, para dar respuesta de manera más efectiva a sus necesidades; 3) Hacer un mejor trabajo de articulación de la investigación y la práctica; 4) Diseminar los resultados de la investigación de manera más amplia.

Inglis y Foster (2018) analizan los artículos publicados en las revistas Educational Studies in Mathematics y Journal for Research in Mathematics Education, fijando la atención en los 
cambios en las orientaciones teóricas que han tenido lugar desde la fundación de estas revistas. Los autores revelan el predominio de los programas de investigación basados en la psicología experimental, el constructivismo y los enfoques socioculturales. También evalúan la medida en que ha tenido lugar el llamado "giro social" en los programas de investigación (LERMAN, 2000) y el decaimiento de las investigaciones experimentales, las cuales, no obstante, continúan publicándose con frecuencia en revistas de psicología o educación. Durante la década de los 90 , el foco de la investigación en educación matemática se desplazó en gran medida desde lo cognitivo a lo social: desde teorías que se centran en los procesos de pensamiento de los individuos a "teorías que ven el significado, el pensamiento, y el razonamiento como productos de la actividad social" (LERMAN, 2000, p. 23). Este cambio revela que la investigación en educación matemática está sujeta a tendencias que introducen sesgos y parcialidades en los temas de investigación. Inglis y Foster (2018) concluyen que "la educación matemática se beneficiaría de una mayor interacción entre la investigación basada en la psicología experimental y la investigación sociocultural" (p. 494).

Otro de los temas al que se concede gran atención en la investigación didáctica es el de los cambios en la educación matemática derivados de los recientes desarrollos tecnológicos, los cuales están desafiando las tradicionales visiones sobre el currículo, la enseñanza, el aprendizaje y la evaluación.

"¿Qué formas de currículo, enseñanza, aprendizaje y evaluación son las más adecuadas teniendo en cuenta la rápida evolución tecnológica? ¿Cómo pueden los profesores seguir el ritmo de los avances $y$, al mismo tiempo, hacer frente a su carga docente, a menudo demasiado pesada? Teniendo en cuenta los últimos avances, ¿cómo deberían ser los planes de estudio de álgebra, geometría y cálculo en el futuro? ¿Cómo deberían ser las pruebas en las matemáticas escolares? ¿Qué ayudas tecnológicas deberían poder utilizar los estudiantes en los exámenes, y qué implicaciones tiene esta cuestión para los responsables de elaborar las políticas de evaluación? (CLEMENTS, 2013, p. viii)

A partir de estas discusiones, se observa que los investigadores tienen que atender a los problemas docentes respondiendo a qué matemáticas enseñar y cómo, de manera sistemática y siguiendo una metodología rigurosa que produzca conocimiento científico. Pero los problemas son abordados asumiendo determinados principios y métodos específicos que caracterizan los marcos teóricos desde los cuales se formulan dichos problemas e interpretan los resultados. Por tanto, es necesario reflexionar sobre la elección y las implicaciones del modelo conceptual que se emplea (aunque sea tácitamente). Como plantea Schoenfeld: "Por ejemplo, ¿qué fenómenos no se tienen 
en cuenta en esta perspectiva? ¿A cuáles se les da una importancia significativa? ¿Cómo pueden esos prejuicios teóricos configurar la interpretación de la situación?" (SCHOENFELD, 2007, p. 80).

En este trabajo nos planteamos emplear herramientas del EOS para diseñar un marco de referencia que permita identificar y clasificar los problemas de investigación en educación matemática, y que conecte tanto la componente científico-teórica como tecnológico-práctica en la investigación. EI EOS es un sistema teórico modular e inclusivo para la educación matemática que incluye principios y herramientas metodológicas para abordar las cuestiones centrales implicadas en los procesos de enseñanza y aprendizaje de las matemáticas, que se detallan en la Sección 3. La unidad básica del análisis didáctico es un proceso de instrucción, entendiendo por instrucción matemática "los procesos de enseñanza y aprendizaje organizados, en los cuales intervienen unos determinados sistemas de prácticas matemáticas (conocimientos institucionales), unos sujetos (estudiantes) cuyo compromiso es la apropiación personal de dichas prácticas, el profesor 0 director del proceso de instrucción y unos recursos instruccionales" (GODINO; CONTRERAS y FONT, 2006, p. 41). En dichos procesos es necesario tener en cuenta seis dimensiones o facetas: epistémica, cognitiva, afectiva, interaccional, mediacional y ecológica (Figura 1). Dichas facetas pueden servir como categorías primarias para clasificar los focos de atención del análisis didáctico, los cuales son refinados a partir de los componentes que proporcionan las diversas herramientas elaboradas en el EOS.

Figura 1. Facetas de un proceso instruccional

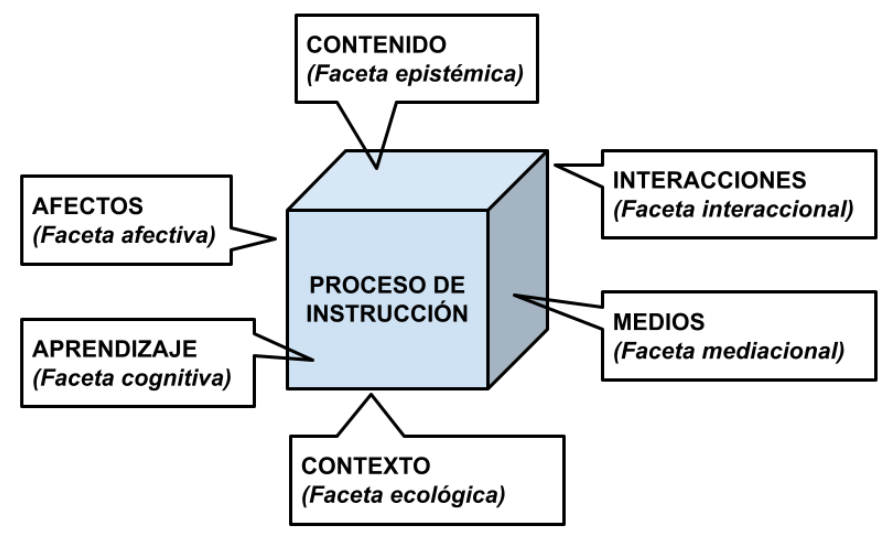

Fuente: Elaboración propia.

En el EOS se define la idoneidad didáctica de un proceso de instrucción como el grado en que éste (o una parte del mismo) reúne ciertas características que permiten calificarlo como óptimo o adecuado para conseguir la adaptación entre los significados personales logrados por los estudiantes (aprendizaje) y los significados institucionales pretendidos o implementados 
(enseñanza), teniendo en cuenta las circunstancias y recursos disponibles (entorno). La idoneidad didáctica es un rasgo graduable que supone la articulación coherente de las siguientes seis facetas (GODINO et al., 2007) relacionadas con las correspondientes del proceso instruccional'1:

- Idoneidad epistémica: grado de representatividad de los significados institucionales implementados (o pretendidos), respecto de un significado de referencia.

- Idoneidad cognitiva: grado en que los significados implementados están en la zona de desarrollo potencial de los estudiantes, así como la proximidad de los significados personales logrados a los significados implementados.

- Idoneidad interaccional: grado en que las configuraciones y trayectorias didácticas permiten identificar conflictos semióticos potenciales (que se puedan detectar a priori), y resolver los conflictos que se producen durante el proceso de instrucción.

- Idoneidad mediacional: grado de disponibilidad y adecuación de los recursos materiales y temporales necesarios para el desarrollo del proceso de enseñanza y aprendizaje.

- Idoneidad afectiva: grado de implicación (interés, motivación, ...) del alumnado en el proceso de instrucción.

- Idoneidad ecológica: grado en que el proceso de instrucción se ajusta al proyecto educativo del centro y la sociedad, así como a los condicionamientos del entorno en que se desarrolla.

A partir de los conocimientos derivados de la investigación considerados pertinentes y adecuados por una amplia mayoría de la comunidad educativa se establecen criterios de idoneidad como referencia para el diseño, implementación y evaluación de procesos de instrucción. Ello es debido a que, como resultado de la investigación didáctica, se obtienen conocimientos que se traducen en criterios o normas de actuación preferente sobre qué matemáticas se deberían enseñar y cómo, según los contextos, circunstancias y personas implicadas.

\section{Tipos de problemas de investigación e investigaciones de diseño}

Dentro del EOS se asume que para responder a la problemática de qué matemáticas enseñar y cómo, de manera científica, es necesario considerar previamente las siguientes cuestiones generales (GODINO et al., 2019):

\footnotetext{
1 Las nociones de significado (institucional, personal y sus diferentes tipos), de conflicto semiótico, así como las de configuración y trayectoria didáctica se pueden consultar en Godino; Batanero y Font (2007).
} 
- Problema epistemológico: ¿Cómo emerge y se desarrolla la matemática?

- Problema ontológico: ¿Qué es un objeto matemático? ¿Qué tipos de objetos intervienen en la actividad matemática?

- Problema semiótico-cognitivo: ¿Qué es conocer un objeto matemático? ¿Qué significa el objeto para un sujeto en un momento y circunstancias dadas?

- Problema educativo-instruccional: ¿Qué es la enseñanza? ¿Qué es el aprendizaje? ¿Cómo se relacionan?

- Problema ecológico: ¿Qué factores condicionan y soportan el desarrollo de los procesos instruccionales y qué normas los regulan?

- Problema de optimización del proceso de instrucción: ¿Qué tipo de acciones y recursos se deberían implementar en los procesos de instrucción para optimizar el aprendizaje matemático?

- Problema de formación de profesores: ¿Qué conocimientos y competencias deberían tener los profesores para optimizar el aprendizaje en los procesos de instrucción en matemáticas?

En Godino et al. (2019) se describen los principios y herramientas metodológicas mediante los cuales se abordan estas cuestiones centrales de la investigación didáctica, entendida como campo de investigación cientifica y tecnológica. Con el enunciado conjunto de estas preguntas se muestra la complejidad de la optimización de los procesos de instrucción, ya que previamente se deben abordar estudios de índole epistemológica, ontológica, cognitiva, semiótica y ecológica. Cada uno de ellos debe, además, ser analizado y descompuesto para que pueda ser investigado con las exigencias metodológicas propias del trabajo científico. Seguidamente presentamos un esquema operativo para definir problemas de investigación que tiene en cuenta las diferentes facetas, las cuales deben constituir focos de interés del análisis didáctico.

\subsection{Criterios de clasificación de los problemas de investigación ${ }^{2}$}

Un primer criterio para la clasificación de los problemas se refiere al contenido matemático sobre el que se centra la investigación. El problema se puede referir al estudio de temas relativos a Aritmética, Geometría, Medida, Álgebra, Estadística, Cálculo, etc. El nivel educativo (educación infantil, primaria, secundaria, universidad, formación de profesores, etc.) al que se refiere el

\footnotetext{
2 En la bibliografía sobre metodología de investigación educativa se pueden encontrar otros criterios para clasificar los problemas y métodos que pueden complementar a los aquí presentados. Por ejemplo, Cohen, Manion y Morrison (2007) describen como "estilos de investigación educativa": investigación naturalista y etnográfica; histórica y documental; estudio de casos, experimental, cuasi-experimental, meta-análisis; investigación acción, entre otros.
} 
proceso de instrucción es otro criterio que permite organizar las cuestiones de investigación. Además del contenido y el nivel educativo, debemos fijar el foco de la investigación, que puede ser uno o varios de los siguientes:

- Epistémico: se investiga el propio contenido matemático y las diferentes formas en que puede presentarse (más o menos formal, más o menos completo) en la actividad matemática.

- Cognitivo: se analiza cómo lo estudiantes aprenden, razonan y entienden las matemáticas, sus estrategias en la resolución de problemas, qué dificultades 0 conflictos semióticos muestran en el proceso de instrucción y cómo progresan en su aprendizaje.

- Afectivo: se centra en los aspectos afectivos, emocionales y actitudinales de los estudiantes con relación a los objetos matemáticos y al proceso de instrucción implementado.

- Interaccional: se analizan los roles del profesor y los estudiantes en la gestión de las tareas, la identificación y resolución de conflictos de aprendizaje, así como el tipo de interacciones que se puede establecer en el aula.

- Mediacional: se analizan los recursos (tecnológicos, materiales y temporales) apropiados para potenciar el aprendizaje de los estudiantes, así como la elaboración de los mismos.

- Ecológico: se centra en las relaciones del contenido matemático con otras disciplinas, así como en los factores curriculares, socio-profesionales, políticos y económicos que condicionan los procesos de instrucción matemática.

El análisis didáctico de los procesos de formación de profesores deberá tener en cuenta las seis facetas mencionadas referidas, en este caso, a los conocimientos didáctico-matemáticos (GODINO; GIACOMONE; BATANERO y FONT, 2017), al desarrollo de las competencias profesionales y al estudio de los factores condicionantes de los mismos.

Dependiendo de los marcos teóricos que se utilicen para centrar el foco de la investigación, atendiendo a las facetas descritas, se pueden aportar elementos que ayudan a su realización de una manera más detallada, lo cual es útil para describir y comprender los procesos instruccionales. 
Por otro lado, la clasificación de los problemas de investigación según su foco se puede complementar con la siguiente tipología, que viene caracterizada por su intencionalidad o finalidad con la cual se realiza:

- Descriptiva de significados, procesos y factores (¿Qué es ...? ¿Cómo es ...?) .

- Explicativa de los procesos de enseñanza y aprendizaje y los efectos de los factores intervinientes (¿Por qué ...? ¿Qué varia?).

- Predictiva o de implementación de acciones para el logro de un fin (¿Cómo diseñar o motivar ...? ¿Qué pasa si cambio ...?).

- Valorativa de la idoneidad de un proceso de instrucción o alguno de sus componentes (¿En qué medida es adecuado o idóneo ...?).

Además, se pueden distinguir las investigaciones por su amplitud, según sean estudios de casos, muestras (que pueden ser probabilísticas o no), o censos (COHEN et al., 2007).

La Figura 2 resume los criterios de clasificación de los problemas de investigación que hemos propuesto. Podemos definir problemas específicos fijando un contenido matemático, una faceta, una finalidad y un grado de generalidad o amplitud; pero también se pueden definir problemas abarcando varias de estas categorías o diferentes contenidos.

Figura 2. Clasificación de los problemas de investigación

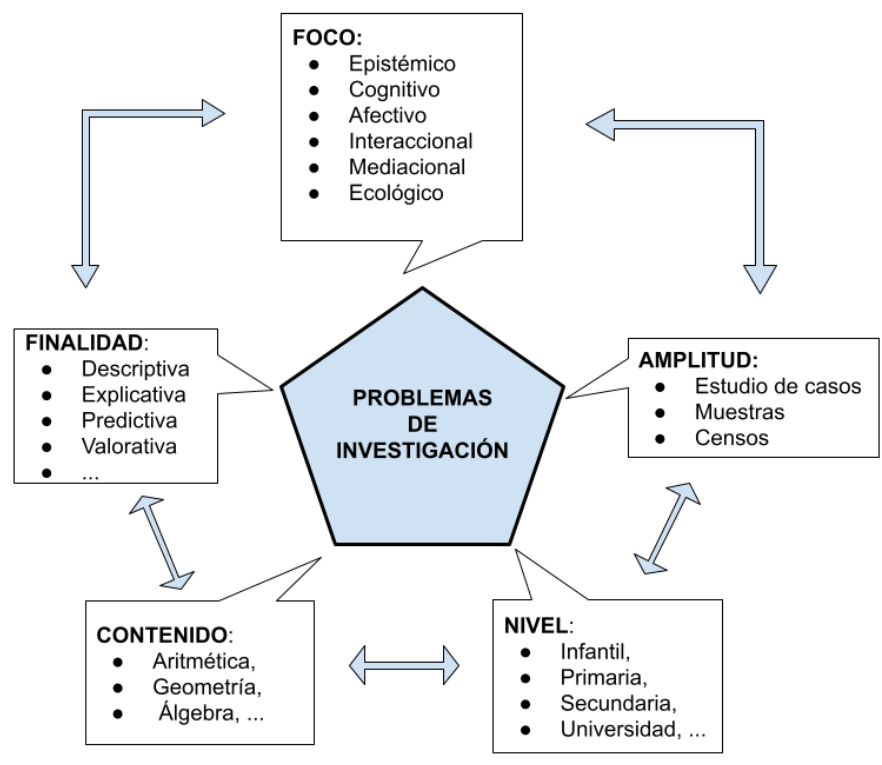

Fuente: Elaboración propia.

Teniendo en cuenta algunos constructos desarrollados en el EOS (GODINO, 2002; GODINO et al., 2007), la Figura 3 muestra los componentes de cada uno de los focos 
anteriormente descritos. En las dimensiones epistémica y cognitiva se distinguen subcomponentes basados en la noción de significado pragmático de los objetos matemáticos así como en la noción de configuración de objetos y procesos que intervienen en las prácticas matemáticas (problemas, elementos lingüísticos, conceptos, proposiciones, procedimientos y argumentos) (GODINO, 2002). Cada una de estos focos, componentes y subcomponentes puede ser el foco de atención del estudio y, por tanto, dan lugar a la formulación de preguntas de investigación específicas. El esquema de la Figura 3 ayuda a detallar los problemas de investigación de una manera sistemática, sin perder de vista su visión de conjunto en torno a las interacciones que pueden plantearse entre las facetas y sus componentes.

Figura 3. Facetas y componentes del proceso instruccional que pueden ser focos de la investigación

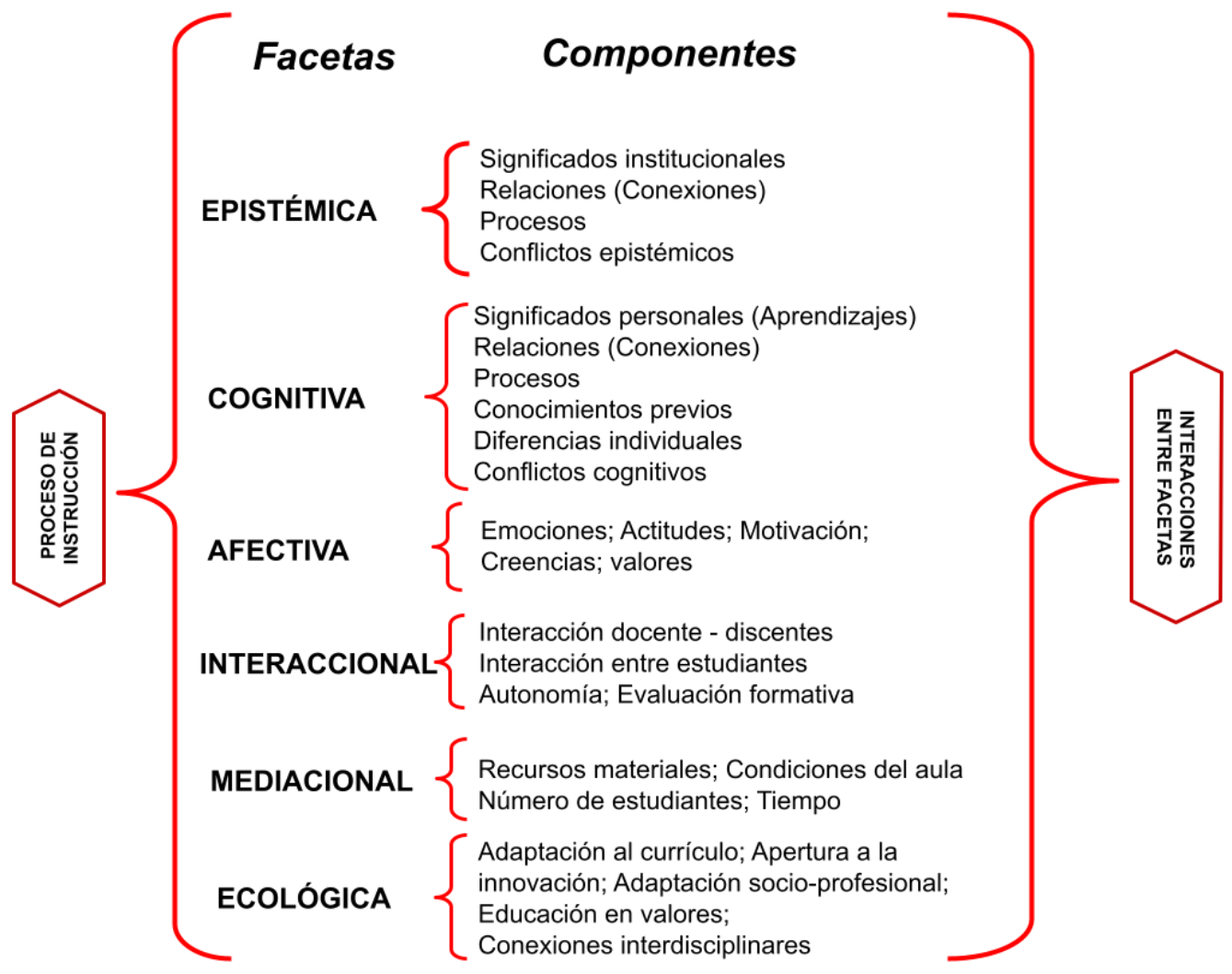

Fuente: Elaboración propia.

Por tanto, con el fin de garantizar la importancia o relevancia de la investigación que se realiza, en el proceso analítico de formulación de los problemas de investigación no se debería perder de vista la globalidad de las facetas, componentes y variables que intervienen en un proceso de instrucción matemática. Así es que destacamos un tipo de investigación que tiene en cuenta las interacciones entre las distintas facetas y componentes como es la investigación de 
diseño instruccional (KELLY; LESH y BAEK, 2008), que describimos con más detalle en el siguiente apartado. Este tipo de investigación tiene una finalidad predictiva, esto es, responde al esquema "Si aplicamos el tratamiento $X$ en unas circunstancias determinadas entonces se obtienen los resultados Y”. En principio, esta investigación está más próxima a las cuestiones y necesidades de la práctica docente, sobre todo porque considera las diferentes facetas y componentes que intervienen en los procesos instruccionales.

El supuesto en que se basa la realización de experimentaciones es que, en condiciones similares a las circunstancias de la experimentación, se obtendrán resultados similares a los de la misma. Esta potencial generalidad de los resultados puede ocurrir no solo en los estudios de tipo cuantitativo sino también en las descripciones ricas y densas de tipo antropológico que se realizan en el campo de las ciencias humanas, donde es difícil implementar todos los requisitos de las investigaciones experimentales o cuasi-experimentales (SCHOENFELD, 2007).

\subsection{Investigaciones educativas basadas en el diseño}

Como se describe en Godino et al. (2013), la investigación basada en el diseño (IBD) (BROWN, 1992; KELLY; LESH y BAEK, 2008) constituye una familia de aproximaciones metodológicas orientadas al estudio del aprendizaje en contexto. Utiliza el diseño instruccional y la investigación sistemática de estrategias y herramientas instruccionales, tratando que sean interdependientes, sobreentendiéndose que la investigación incluye no solo la fase de diseño, sino también la experimentación en contextos de clase y la evaluación de resultados. Trata de superar la brecha entre las investigaciones científicas desligadas de la práctica educativa y las innovaciones realizadas de manera poco, o nada rigurosas. Asume que la investigación educativa separada de la práctica puede no tener en cuenta la influencia de los contextos sobre la naturaleza compleja de los resultados, o no identificar adecuadamente las restricciones y factores condicionantes. En educación matemática, las IBD se realizan aplicando diferentes teorías de base en los diseños e interpretación de los resultados: en Bakker y van Eerde (2015) se describe una investigación de diseño basada en los supuestos de la educación matemática realista; Artigue (2015) describe la metodología de la ingeniería didáctica como un tipo de IBD, basada en la teoría de situaciones didácticas, o en la teoría antropológica de lo didáctico; Henrick, Cobb y Jackson (2015) describen una IBD de la que se deriva una teoría de acción para la mejora de la instrucción matemática en sistemas escolares a gran escala, apoyada en la teoría fundamentada (grounded theory). 
La mayoría de estas investigaciones se conceptualizan como estudios de casos orientados a apoyar el aprendizaje de grupos de estudiantes en un dominio de contenido particular. La intención teórica es identificar y describir patrones en el razonamiento de los estudiantes y relacionarlos con los medios utilizados para apoyar y organizar su desarrollo. Como hemos indicado, la problemática de la IBD consiste en elaborar recursos instruccionales para la mejora de la enseñanza y aprendizaje de las matemáticas, en contextos escolares naturalistas, basados en la investigación. Estas investigaciones se llevan a cabo distinguiendo tres fases en los experimentos (COBB y GRAVEMEIJER, 2008): 1) Preparación del experimento; 2) Experimentación para apoyar el aprendizaje; 3) Análisis retrospectivos de los datos generados durante la realización del experimento. Se aborda, por tanto, un problema predictivo, pero previamente fundamentado en respuestas a cuestiones de tipo descriptivo y explicativo.

Se puede asumir que las investigaciones de diseño son más próximas a la práctica de la enseñanza, ya que, usualmente, tienen en cuenta la riqueza y complejidad de factores que condicionan la práctica. Sin embargo, dado que el conocimiento que aporta este tipo de investigación es predictivo, no se pueden derivar de ella valoraciones y normas de acción, como se requiere en la intervención eficiente sobre la práctica. La superación de la brecha existente entre el conocimiento científico y la práctica de la enseñanza requiere el desarrollo de una teoría que explicite el sistema de principios axiológicos, así como los criterios valorativos y normativos sobre la acción educativa eficiente derivados de la investigación científica. En el marco del EOS esta interfaz viene dada por la Teoría de la Idoneidad Didáctica que describimos en la siguiente sección.

\section{La idoneidad didáctica como nexo entre la investigación teórica y la práctica de la enseñanza}

El EOS aborda el problema tecnológico de optimización del proceso de instrucción mediante investigaciones del tipo IBD (diseño educativo) con finalidad predictiva. Los resultados de estas investigaciones, interpretados desde el marco teórico del EOS, dan respuestas tecnológicas a la problemática docente sobre "qué enseñar y cómo" en circunstancias locales. No obstante, dichas investigaciones se basan en las respuestas a los problemas previos de tipo descriptivo y explicativo implicados, propios de la investigación básica o fundamental (Figura 4). Como se indica en esta figura, entre los problemas predictivos propios de las investigaciones de diseño y el problema docente hay una separación que debe ser salvada asumiendo unos principios y valores coherentes con los fines educativos fijados en el currículo. Dado que los fines educativos y los currículos pueden ser diversos, se abre aquí un área problemática de indagación que 
describimos en la Figura 4 como problemas valorativos ${ }^{3}$, para los cuales se propone en el EOS la herramienta de la idoneidad didáctica. Se trata de la realización de meta-análisis para identificar principios, valores y medios asumidos por la investigación básica y aplicada sobre los cuales haya un cierto grado de consenso para ser aplicados en la práctica educativa.

Figura 4. Conexiones entre tipos de problemas de investigación

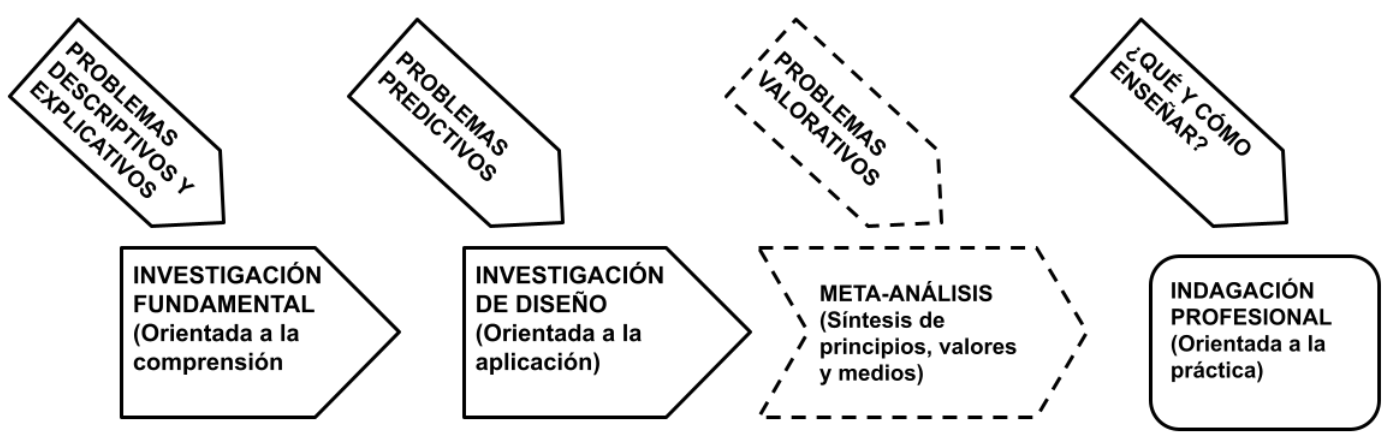

Fuente: Elaboración propia.

La noción de idoneidad didáctica ha sido introducida dentro del EOS como herramienta de paso desde una didáctica descriptiva-explicativa a una didáctica normativa, esto es, orientada hacia la intervención efectiva en el aula (GODINO; CONTRERAS y FONT, 2006). Por ello, puede servir de punto de partida para una teoría de diseño instruccional (Teoría de la Idoneidad Didáctica, TID) que tenga en cuenta, de manera sistémica, las dimensiones epistémica - ecológica, cognitiva - afectiva e interaccional - mediacional implicadas en los procesos de instrucción de las áreas curriculares específicas.

"Por criterio de idoneidad se debe entender una norma de corrección que establece cómo debería realizarse un proceso de enseñanza y aprendizaje. Estos criterios deben ser entendidos como normas de corrección emanadas del discurso argumentativo de la comunidad científica, cuando éste está orientado a conseguir un consenso sobre lo que se puede considerar como mejor." (BREDA; FONT y PINO-FAN, 2018, p. 264).

Se parte de la base de que en las ciencias sociales y educativas es posible formular criterios de idoneidad, en la forma de juicios de valor ${ }^{4}$, "Se debería hacer esto y no aquello", en aquellas circunstancias en que dichos juicios de valor tienen carácter social y es posible explicitar

\footnotetext{
3 El problema valorativo se puede relacionar con la elección del paradigma didáctico de referencia (GASCÓN y NICOLÁS, 2021), que incluye además de un modelo epistemológico de referencia, la formulación de los fines educativos que propugna.

4 El principio de Weber (1917/2010) sobre la neutralidad ética del científico dice que "no hay lugar en la ciencia para los juicios de valor". Pero diversos autores sostienen que "en la ciencia hay un lugar para los valores sociales, impersonales y objetivos y juicios de valor, con la exigencia de una prueba lógica y/o empírica" (RUGINA, 1998, p. 817).
} 
un fundamento para su formulación. Conllevan una racionalidad, por lo que pueden ser objeto de escrutinio científico (BUNGE, 1999; RUGINA, 1998).

Pensamos que el universo de conocimientos didáctico-matemáticos, y por tanto también el de los criterios de idoneidad, se puede categorizar de manera jerárquica distinguiendo entre criterios generales (los ligados a cada una de las seis dimensiones), criterios parciales, vinculados a los distintos componentes propuestos para cada faceta, y criterios específicos, relacionados con aspectos particulares de los procesos de instrucción de contenidos. Por ejemplo, un criterio específico para la enseñanza de la proporcionalidad, sería dar oportunidad a los estudiantes de distinguir las situaciones multiplicativas de las aditivas, y evitar el aprendizaje algorítmicomemorístico de la regla de tres. Las normas de idoneidad, por tanto, pueden tener en unos casos el carácter de principio, como son las relativas a las formuladas en términos generales para las dimensiones, o en otros verse como reglas, por ejemplo, las normas relacionadas con el aprendizaje de contenidos específicos. Una norma de idoneidad que tiene un claro carácter de regla podría ser, "el proceso de instrucción deberá evitar la transmisión de conocimientos erróneos".

El cumplimiento en cada caso de los criterios de idoneidad lleva asociado diversos indicadores empíricos. Por ejemplo, se asume que la comprensión y competencia matemática de los estudiantes está ligada al uso de diversas representaciones, su tratamiento y conversión. En consecuencia, se asume que un proceso de instrucción deberá procurar (norma) que los estudiantes tengan oportunidades de uso de diferentes sistemas de representación, su conversión y tratamiento. La emisión de un juicio sobre la mayor o menor idoneidad de un proceso instruccional específico se basará en la observación de indicadores empíricos sobre el uso de los sistemas de representación que se hace o propone.

En las Tablas 1 y 2 incluimos la formulación de criterios de idoneidad para las dimensiones epistémica y cognitiva y sus respectivos componentes 5 . El modelo presentado en estas tablas desarrolla y reorganiza el sistema de facetas y componentes descrito en Godino (2013) y el propuesto por Breda et al. (2018), en cuanto a las dimensiones epistémica y cognitiva, destacando el papel central que desempeña la noción de significado pragmático, tanto desde el punto de vista institucional (epistémico), como personal (cognitivo). También se enfatiza el papel de los procesos

\footnotetext{
5 Consideramos necesario distinguir entre facetas y componentes de un proceso de instrucción respecto de los criterios e indicadores de idoneidad. El criterio es una norma (en unos casos como principio, en otros incluso como regla) que se debería seguir para lograr que un proceso de instrucción tenga una alta idoneidad; el indicador es la manifestación observable que se tiene como resultado de la aplicación del criterio; las facetas y sus componentes son categorías o agrupaciones de los criterios.
} 
en la instrucción matemática. La Tabla 3 incluye los criterios generales de idoneidad para las restantes facetas de la idoneidad6. En Godino (2013) se describen algunas teorías y enfoques curriculares que comparten los principios enunciados para los indicadores de la idoneidad de los componentes de las diferentes facetas.

Tabla 1. Criterios de idoneidad para la faceta epistémica y sus componentes

\begin{tabular}{|c|c|}
\hline Faceta & Criterios según componentes \\
\hline \multirow{8}{*}{$\begin{array}{l}\text { Epistémica } \\
\text { (Representatividad) } \\
\text { Los significados } \\
\text { institucionales del } \\
\text { contenido y las } \\
\text { configuraciones de } \\
\text { objetos y procesos } \\
\text { implementados deberían } \\
\text { ser representativos del } \\
\text { significado global de } \\
\text { referencia, teniendo en } \\
\text { cuenta las circunstancias } \\
\text { contextuales y personales } \\
\text { de los sujetos implicados. }\end{array}$} & Significados \\
\hline & $\begin{array}{l}\text { Se deberían tener en cuenta los diversos objetos primarios } \\
\text { implicados en la actividad matemática (situaciones, lenguajes, } \\
\text { conceptos y propiedades, procedimientos y argumentos) que } \\
\text { conforman los significados parciales del contenido, seleccionando } \\
\text { aquel o aquellos cuyo estudio se adapta a las circunstancias } \\
\text { contextuales y personales de los sujetos implicados. Todo ello ha } \\
\text { de estar contextualizado en situaciones comprensibles para el } \\
\text { estudiante. }\end{array}$ \\
\hline & Relaciones (conexiones) \\
\hline & $\begin{array}{l}\text { Se deberían relacionar entre sí los significados parciales estudiados } \\
\text { y los objetos que intervienen en las prácticas correspondientes, así } \\
\text { como con el contenido de otros temas que el estudiante ya conoce. }\end{array}$ \\
\hline & Procesos \\
\hline & $\begin{array}{l}\text { Se debería tener en cuenta la diversidad de procesos (secuencias } \\
\text { de prácticas) de los cuales emergen los objetos que intervienen en } \\
\text { las prácticas matemáticas (problematización, representación, } \\
\text { definición, generalización, modelización, ...). }\end{array}$ \\
\hline & Conflictos epistémicos \\
\hline & $\begin{array}{l}\text { Se debería evitar las discordancias entre los significados de los } \\
\text { objetos y procesos implementados y los correspondientes a la } \\
\text { institución de referencia (ausencia, además, de errores y } \\
\text { ambigüedades). }\end{array}$ \\
\hline
\end{tabular}

Fuente: Elaboración propia.

El diseño de un proceso de instrucción con alta idoneidad epistémica comienza con la reconstrucción de un significado global de referencia del contenido para el que se planifica 0 implementa su enseñanza, el cual articula los diversos significados parciales del mismo. Los significados son entendidos de manera pragmática, esto es, como sistemas de prácticas operativas y discursivas que se realizan para dar respuesta a una clase de situaciones-problemas en las que el objeto cobra significado (GODINO; BURGOS y GEA, 2021). Ejemplos de la reconstrucción del significado global de algunos objetos matemáticos se describen en Batanero y Díaz (2007), Wilhelmi, Godino y Lacasta (2007), entre otros trabajos.

\footnotetext{
6 En Godino (2013) se enuncian indicadores de idoneidad para los diferentes subcomponentes de las facetas epistémica y cognitiva; en las Tablas 1 y 2 complementamos ese trabajo con la formulación de criterios (principios 0 normas) más generales para las propias facetas epistémica y cognitiva y sus respectivas componentes.
} 
En un proceso de instrucción específico hay que tener en cuenta las circunstancias contextuales y personales de los sujetos implicados, por lo que es inevitable centrar la atención en una parte del significado global. Además, dado que la matemática no solo es una trama de objetos histórica y culturalmente construidos, sino también una actividad de las personas, de la que emergen dichos objetos, la instrucción debe tener en cuenta los procesos matemáticos implicados (problematización, representación, definición, generalización, modelización, ...). En consecuencia, asumiendo como principio axiológico el proponer o implementar unas "buenas matemáticas", se sigue que los significados seleccionados deben ser representativos del significado global y ofrecer riqueza de procesos en su desarrollo.

Tabla 2. Criterios de idoneidad para la dimensión cognitiva y sus componentes

\begin{tabular}{|c|c|}
\hline Faceta & Criterios según componentes \\
\hline \multirow{2}{*}{$\begin{array}{l}\text { Cognitiva } \\
\text { (Proximidad y reto } \\
\text { alcanzable) }\end{array}$} & $\begin{array}{l}\text { Significados personales (Aprendizajes) } \\
\text { Se debería lograr que los significados personales construidos por los } \\
\text { estudiantes se correspondan con los significados institucionales } \\
\text { pretendidos o implementados. } \\
\text { La evaluación de los aprendizajes logrados debería tener en cuenta las } \\
\text { características personales de los estudiantes y los distintos niveles de } \\
\text { comprensión y competencia que pueden alcanzar. La evaluación debería } \\
\text { servir para mejorar el proceso instruccional. }\end{array}$ \\
\hline & $\begin{array}{l}\text { Relaciones (conexiones) } \\
\text { El aprendizaje debería ser de tipo relacional, de modo que los estudiantes } \\
\text { sean capaces de comprender y relacionar los distintos significados } \\
\text { incluidos en el proceso de enseñanza y objetos implicados. }\end{array}$ \\
\hline \multirow{4}{*}{$\begin{array}{l}\text { Los objetivos de } \\
\text { aprendizaje } \\
\text { deberían suponer } \\
\text { un reto cognitivo } \\
\text { alcanzable para } \\
\text { los estudiantes, } \\
\text { teniendo en } \\
\text { cuenta sus } \\
\text { circunstancias } \\
\text { contextuales y } \\
\text { personales. }\end{array}$} & $\begin{array}{l}\text { Procesos } \\
\text { Se debe tener en cuenta la competencia del estudiante para implementar } \\
\text { procesos matemáticos específicos del contenido (modelización, } \\
\text { generalización, resolución o planteamiento de problemas, prueba, } \\
\text { representación ...) y metacognitivos (reflexión sobre los propios procesos } \\
\text { de pensamiento matemático). }\end{array}$ \\
\hline & $\begin{array}{l}\text { Conocimientos previos } \\
\text { El proceso de instrucción debería considerar los conocimientos previos } \\
\text { que tienen los estudiantes a los que va dirigido para abordar el estudio del } \\
\text { contenido pretendido. }\end{array}$ \\
\hline & $\begin{array}{l}\text { Diferencias individuales } \\
\text { El proceso de instrucción debería apoyar a los estudiantes según sus } \\
\text { diferencias individuales en conocimientos previos y estilos de aprendizaje } \\
\text { en el proceso de estudio del contenido pretendido o implementado. }\end{array}$ \\
\hline & $\begin{array}{l}\text { Conflictos cognitivos } \\
\text { El proceso de instrucción debería poder identificar en los estudiantes los } \\
\text { conflictos cognitivos que la investigación didáctica ha revelado como } \\
\text { propios y característicos del contenido pretendido o implementado y } \\
\text { ayudarles a superarlos. }\end{array}$ \\
\hline
\end{tabular}

Fuente: Elaboración propia. 
El criterio o norma general de idoneidad cognitiva está inspirado en el concepto de zona de desarrollo potencial (VYGOTSKY, 1934), por lo que los objetivos de aprendizaje deben implicar el desarrollo de conocimientos y competencias matemáticas valiosas que impliquen un esfuerzo alcanzable con el apoyo del profesor, los compañeros, los conocimientos previos y capacidades individuales (principio de equidad). Se asume un aprendizaje relacional y con comprensión de los significados institucionales.

Tabla 3. Criterios de idoneidad en las restantes facetas ${ }^{7}$

\begin{tabular}{|c|l|}
\hline Facetas & \multicolumn{1}{|c|}{ Criterios } \\
\hline Afectiva & $\begin{array}{l}\text { Implicación } \\
\text { El proceso de instrucción debería lograr el mayor grado posible de implicación } \\
\text { del alumnado (interés, motivación, autoestima, disposición, . . . ). }\end{array}$ \\
\hline Interaccional & $\begin{array}{l}\text { Negociación } \\
\text { Las configuraciones y trayectorias didácticas que se implementen deberían } \\
\text { permitir identificar los conflictos semióticos potenciales y poner los medios } \\
\text { adecuados para su resolución. }\end{array}$ \\
\hline Mediacional & $\begin{array}{l}\text { Disponibilidad } \\
\text { Se debería disponer de los recursos materiales y temporales adecuados para } \\
\text { el desarrollo óptimo del proceso de enseñanza y aprendizaje. }\end{array}$ \\
\hline Ecológica & $\begin{array}{l}\text { Adaptación } \\
\text { El proceso de instrucción debería estar en concordancia con el proyecto } \\
\text { educativo del centro y la sociedad, teniendo en cuenta los condicionamientos } \\
\text { del entorno en que se desarrolla y las innovaciones basadas en la } \\
\text { investigación educativa. }\end{array}$ \\
\hline
\end{tabular}

Fuente: Elaboración propia.

Respecto al criterio de idoneidad interaccional es necesario tener en cuenta la complejidad ontosemiótica del conocimiento matemático y su naturaleza sociocultural. En consecuencia, podría ser más idóneo implementar un modelo didáctico mixto basado en la colaboración entre el profesor y los estudiantes, que supere el dilema entre la transmisión del conocimiento y la indagación por los propios estudiantes (GODINO y BURGOS, 2020).

Con el sistema de criterios de idoneidad sintetizados en las tablas 1, 2 y 3 se trata de promover la enseñanza y aprendizaje de unas matemáticas ricas en significados y procesos, local y personalmente optimizados en sus diferentes componentes. Este modelo de instrucción matemática concuerda con las características de una "enseñanza ambiciosa" (LAMPERT et al., 2010) y el desarrollo de la "proficiencia matemática" (KILPATRICK; SWAFFORD, y FINDELL, 2001; RAND MATHEMATICS STUDY PANEL, 2003), "en la que la comprensión conceptual,

\footnotetext{
7 Ver Godino (2013) para indicadores de idoneidad de las respectivas componentes de las facetas afectiva, interaccional, mediacional y ecológica.
} 
fluencia procedimental, competencia estratégica y disposición productiva, están entrelazadas en la práctica matemática y el aprendizaje en todos los niveles y todos los estudiantes" (LAMPERT et al., 2010, p. 129).

El sistema de criterios e indicadores de idoneidad para las distintas facetas y componentes que intervienen en los procesos de instrucción es un instrumento que permite valorar y mejorar cada fase de un diseño instruccional y por tanto se convierte en un instrumento de mejora de las investigaciones basadas en diseño. En particular, es un recurso en la formación de profesores, quienes deben ser capaces de reflexionar sobre su propia práctica educativa, para tomar decisiones fundamentadas de mejora. En este sentido, en diversas investigaciones sobre formación de profesores de matemáticas realizadas en el marco del EOS, se vienen empleando los criterios e indicadores de idoneidad didáctica, enriquecidos con los resultados de la investigación para guiar su reflexión sobre diversos aspectos de los procesos de instrucción matemática (ARTEAGA; BATANERO y GEA, 2017; BREDA; FONT y LIMA, 2015; BURGOS; BELTRÁN-PELLICER y GODINO, 2020; GEA; BATANERO; ARTEAGA y ESTEPA, 2019; GIACOMONE; GODINO y BELTRÁN-PELLICER, 2018; HUMMES; FONT y BREDA, 2019).

\section{Ejemplos de cuestiones de investigación}

El análisis global de los procesos de instrucción matemática debe realizarse en función del contexto, la institución y nivel educativo en que se desarrolla. El planteamiento de un problema de investigación en didáctica de la matemática, por ejemplo el de la enseñanza y el aprendizaje de la proporcionalidad en educación secundaria, supone una interpretación del ámbito de la actividad matemática que está en juego, es decir, fijar un modelo ontológico de la actividad matemática que permita describir en términos operativos el significado del objeto matemático, en nuestro caso la proporcionalidad, tanto desde el punto de vista institucional (epistémico) como personal (cognitivo).

En este apartado incluimos el enunciado de algunas cuestiones de investigación ilustrativas para las facetas epistémica y cognitiva.

\subsection{Faceta epistémica (significados institucionales)}

Desde la perspectiva epistémica, esto es, del conocimiento matemático institucionalizado, es necesario tener en cuenta la diversidad de significados y su articulación progresiva, atendiendo a los diferentes grados de generalidad y formalización. Para ello, en el marco del EOS, se comienza por determinar el significado institucional de referencia que se va a utilizar para el tema; por ejemplo, puede ser el establecido en el currículo para un cierto nivel, identificando los 
significados parciales y sus relaciones (conexiones). Seguidamente, se aborda el análisis de los significados institucionales planificados, implementados y evaluados (GODINO et al., 2007).

Para la caracterización de los significados institucionales que sirvan de referencia global para el diseño curricular, el EOS propone fijar la atención, en primer lugar, en el análisis ontosemiótico de las prácticas matemáticas. Se comienza con la selección de las situacionesproblemas características del objeto (en particular, aquellas que históricamente lo hicieron surgir) y las distintas maneras de abordar su resolución, en las cuales interviene el objeto bajo estudio de manera crítica. En las prácticas operativas y discursivas que se deben realizar para resolver tales situaciones intervienen otros objetos (conceptos, procedimientos, lenguajes, proposiciones y sus argumentos), así como procesos que ponen en juego diferentes grados de generalidad y formalización. Este análisis permite definir significados parciales del objeto, establecer relaciones jerárquicas entre los mismos en función de su complejidad e interdependencia y, finalmente, identificar conflictos epistémicos (desajustes entre significados de tipo institucional, por ejemplo, entre el significado de referencia y el implementado en un libro de texto o por un profesor).

Las siguientes pueden ser algunas de las cuestiones de tipo epistémico que se pueden formular con finalidad descriptiva, por ejemplo, sobre el contenido específico de la proporcionalidad8:

- ¿Cuáles son las características del significado de referencia de la proporcionalidad en educación secundaria?

- ¿Cuáles son las características del significado institucional planificado/ implementado para la proporcionalidad?

- ¿Podemos identificar significados parciales o diferenciados? ¿Qué relaciones se establecen entre estos significados y sus configuraciones (representaciones, elementos regulativos, argumentos)?

- ¿Qué procesos matemáticos se tienen en cuenta y cómo en el proceso de instrucción planificado / implementado?

- ¿Se observan conflictos epistémicos en el proceso de instrucción planificado / implementado? ¿Cómo habría que evitarlos?

\footnotetext{
${ }^{8}$ En Burgos y Godino (2020) se presenta un modelo ontosemiótico de referencia de la proporcionalidad y sus implicaciones para la planificación curricular en primaria y secundaria.
} 
Fijada la atención en un significado parcial se pueden formular cuestiones específicas para los distintos subcomponentes. Por ejemplo,

- ¿Qué tipos de situaciones-problemas (contextualización, ejercitación, aplicación) han sido implementadas para dotar de significado al contenido?

- ¿Qué modos de expresión (verbal, gráfico, simbólico...), traducciones y conversiones entre los mismos se han implementado?

- ¿Qué definiciones, enunciados y procedimientos se han implementado para sistematizar los conocimientos básicos del tema?

- ¿Qué tipo de justificaciones de las proposiciones y procedimientos se han implementado?

Cuestiones con finalidad explicativa, predictiva y valorativa en la faceta epistémica serían:

- ¿Por qué se enfatiza el uso rutinario de la regla de tres en secundaria en detrimento del significado algebraico-funcional?

- ¿Cómo articular los diversos significados de la proporcionalidad en secundaria para que los estudiantes comprendan y resuelvan las situaciones-problemas?

- ¿Cuál es la idoneidad epistémica del proceso de instrucción implementado sobre el tema?

\subsection{Faceta cognitiva (significados personales)}

Con la dimensión cognitiva nos referimos al desarrollo de los significados personales (aprendizajes), su estado inicial y evolución como consecuencia del proceso instruccional. La herramienta configuración ontosemiótica facilita la descripción pormenorizada de las prácticas matemáticas involucradas en la solución de una situación-problema (GODINO et al., 2007). Cuando tal configuración se aplica al análisis de prácticas, objetos y procesos implicados en la actividad matemática desarrollada por un estudiante para resolver un problema, la configuración será de tipo cognitivo y describirá un significado personal parcial. Sin embargo, como propone el modelo de análisis de la Figura 3, el análisis didáctico-matemático en la faceta cognitiva, además del estudio de los significados personales, requiere contemplar los conocimientos iniciales, los conflictos cognitivos (disparidades entre el significado de referencia y el manifestado por un sujeto) y las diferencias individuales. Por tanto, particularizado a nuestro ejemplo, algunas cuestiones con finalidad descriptiva pueden ser las siguientes: 
- ¿Qué conocimientos y competencias tienen los estudiantes antes de iniciar el estudio de la proporcionalidad en secundaria?

- ¿Existen diferencias relevantes en los conocimientos y competencias de los estudiantes al inicio del estudio del tema?

- ¿En qué medida son capaces los estudiantes de resolver los tipos de problemas de proporcionalidad seleccionando los procedimientos adecuados y justificando las soluciones? ¿Qué estrategias utilizan? ¿Dependen del tipo de problema?

- ¿Cuáles son los conflictos cognitivos que han manifestado los estudiantes durante el proceso de estudio de la proporcionalidad?

- ¿Qué instrumentos de evaluación se podría usar o elaborar para identificar los aprendizajes logrados por los estudiantes? ¿Cómo construirlos?

Por otro lado, algunas cuestiones con finalidad explicativa, predictiva y valorativa serían:

- ¿Qué factores explican las características de los significados personales de los alumnos (dificultades, sesgos, conflictos)?

- ¿Cuáles serían los efectos sobre el aprendizaje de los estudiantes de la aplicación de una secuencia de actividades determinada de ampliación y refuerzo?

- ¿Cuál es el grado de idoneidad cognitiva del proceso de instrucción implementado? ¿Qué elementos se puede cambiar y cómo para aumentar dicha idoneidad?

\section{Enfoque metodológico}

Además de una agenda de investigación, en un marco teórico es importante la discusión metodológica. En educación matemática, y otros campos de investigación en ciencias sociales, se observa un fuerte interés por el empleo de métodos cualitativos de investigación, aunque esto no supone el olvido de los métodos cuantitativos cuando se pretende proponer resultados más ampliamente generalizables. Se está reconociendo la complejidad de los problemas que se abordan en la investigación en ciencias sociales y la necesidad de adoptar una perspectiva pragmatista sobre el uso de metodologías mixtas que permitan comprender las actividades educativas en el contexto en que tienen lugar y al mismo tiempo se aporten recomendaciones generalizables que apoyen la toma de decisiones de política educativa. La perspectiva pragmatista asume la posición de usar una amplia variedad de métodos que deben ser aplicados para abordar cuestiones de investigación complejas. En un mismo proyecto de investigación se puede aplicar métodos cualitativos y cuantitativos con una planificación cuidadosa y reconociendo la potencial 
contribución de cada aproximación (HART et al., 2009; JHONSON y ONWUEGBUZIE, 2004). Además, es importante tener en cuenta la interconexión que existe entre el enunciado de las preguntas y los métodos con los principios y herramientas conceptuales del marco teórico en que se plantea el proyecto de investigación. "Las metodologías son parte de los marcos teóricos usados en la investigación, y por tanto profundamente conectadas con los principios de la teoría y las cuestiones paradigmáticas" (BIKNER-AHSBAHS; KNIPPING y PRESMEG, 2015, p. 533).

\subsection{El análisis ontosemiótico como técnica para determinar significados}

La cuestión epistemológica, esto es, la descripción de cómo emerge y se desarrolla el conocimiento matemático, desde el punto de vista institucional, se investiga en el EOS según el siguiente principio metodológico:

La génesis institucional del conocimiento matemático se investiga mediante: 1) la identificación y categorización de las situaciones-problemas que requieren una respuesta en la que intervenga el objeto, para lo cual, en ocasiones es también necesario un estudio histórico; 2) la descripción de las secuencias de prácticas que se ponen en juego en la resolución.

Como hemos mencionado en las secciones 5.1 y 5.2 , para abordar los problemas de la naturaleza de los objetos matemáticos y su conocimiento se aplica la herramienta configuración ontosemiótica de prácticas, objetos y procesos, en sus versiones duales, epistémica (significados institucionales) y cognitiva (significados personales). El análisis ontosemiótico, esto es, la caracterización de los sistemas de prácticas, los objetos intervinientes en las mismas y de las funciones semióticas que se establecen entre los objetos, da respuesta a los problemas ontológico y semiótico-cognitivo de la educación matemática, permitiendo describir los conocimientos institucionales y personales (GODINO, 2002; GODINO et al., 2011).

Por una parte, y a un nivel que podemos calificar de microscópico, permite identificar significados puestos en juego en una actividad matemática puntual, como es el uso de términos y expresiones. A un nivel más general o macroscópico, permite describir la estructura semiótica de una organización matemática compleja implementada en un proceso de estudio particular o por un estudiante al trabajar con una tarea o a lo largo de un proceso de instrucción (FONT y CONTRERAS, 2008; BURGOS et al., 2021). En ambos niveles, el análisis ontosemiótico permite identificar discordancias o disparidades entre los significados atribuidos a las expresiones por dos sujetos (personas o instituciones) en interacción didáctica. Estos conflictos semióticos pueden explicar, al menos parcialmente, las dificultades potenciales de los alumnos en el proceso de instrucción, así como identificar las limitaciones de las competencias y comprensiones matemáticas efectivamente puestas en juego. La información obtenida con este análisis es 
necesaria si se desea abordar con criterios rigurosos el diseño e implementación del proceso de instrucción y determinar los recursos materiales y de tiempo necesarios.

\subsection{Herramientas metodológicas para el problema educativo-instruccional}

Para abordar el problema educativo-instruccional, esto es, la indagación sobre la enseñanza, el aprendizaje, de cómo se relacionan entre sí, y la identificación de los factores condicionantes para la optimización de los procesos instruccionales, se han desarrollado en el EOS varias herramientas metodológicas específicas, en particular la noción de configuración didáctica (GODINO; CONTRERAS y FONT, 2006). En toda configuración didáctica se diferencian tres componentes: a) una configuración epistémica (sistema de prácticas, objetos y procesos matemáticos institucionales requeridos en la tarea), b) una configuración instruccional (sistema de funciones docentes, discentes y medios instruccionales que se utilizan, así como las interacciones entre los distintos componentes) y c) una configuración cognitiva - afectiva (sistema de prácticas, objetos y procesos matemáticos personales que describe el aprendizaje y los componentes afectivos que le acompañan). La secuencia de configuraciones didácticas, o trayectoria didáctica, da cuenta de la articulación de las distintas configuraciones y su evolución temporal.

Respecto de la identificación de factores condicionantes de los procesos instruccionales en el EOS se dispone de la herramienta metodológica, dimensión normativa. Se trata de tener en cuenta las normas, hábitos y convenciones, generalmente implícitos, que regulan el funcionamiento de la clase de matemáticas y que condicionan en mayor o menor medida los conocimientos que construyen los estudiantes. En Godino et al. (2009) se aborda el estudio sistemático y global de los factores y las normas referidas a las facetas que se deben tener en cuenta en el análisis de los procesos de instrucción (Figura 1). En D’ Amore, Font y Godino (2007) se aborda el estudio sistemático de la dimensión meta-didáctica en los procesos de instrucción matemática.

En cuanto a la cuestión de la optimización de los procesos instruccionales se ha desarrollado la herramienta metodológica de la idoneidad didáctica descrita en la Sección 4. En Godino et al. (2014) se puede ver la aplicación de las diversas nociones teóricas del EOS para abordar el problema educativo-instruccional en su globalidad con una interpretación de la metodología de la ingeniería didáctica, entendida en el sentido generalizado que se describe en Godino, et al. (2013).

\subsection{Metodología para la evaluación de los conocimientos matemáticos}

El problema de la evaluación de los conocimientos matemáticos es planteado por Wheeler 
(1993) desde su dimensión epistemológica: Si necesitamos evaluar el conocimiento matemático de los estudiantes para una multiplicidad de fines, la primera cuestión que debe dilucidarse se refiere a la naturaleza del propio conocimiento. La razón que da este autor nos parece obvia: "¿Cómo podemos evaluar lo que no conocemos?" (p. 87). Esta problemática se corresponde en el EOS con la caracterización de significados. Precisamente, una de las finalidades de la epistemología del conocimiento matemático que propone el EOS es proporcionar criterios para la elaboración de una teoría de la evaluación del mismo, pero previamente se necesita adoptar o elaborar una teoría sobre su naturaleza, variedad y estructura.

La determinación de los conocimientos personales precisa necesariamente de procesos de inferencia, a partir de los conjuntos de prácticas observadas en la situación de evaluación, cuya validez y fiabilidad hay que garantizar ${ }^{9}$. La complejidad de este proceso de inferencia se deduce del hecho de que no solo existen interrelaciones entre los conocimientos referidos a diferentes objetos matemáticos, sino que, incluso para un objeto matemático dado, el conocimiento de un sujeto sobre el mismo, no puede reducirse a un estado dicotómico (conoce o no conoce) ni a un grado o porcentaje unidimensional (conoce $X$ por ciento), lo que hace difícil aplicar a la evaluación de los conocimientos las teorías clásicas psicométricas de maestría de dominio o del rasgo latente (SNOW y LOHMAN, 1991; WEBB, 1992).

El carácter observable de las prácticas sociales permite, mediante un estudio fenomenológico y epistemológico realizado adecuadamente, determinar, para un objeto dado, el campo de problemas asociado, así como los significados institucionales, concretados en las correspondientes configuraciones epistémicas. El análisis de las variables didácticas del campo de problemas proporciona un criterio para estructurar la población de las posibles tareas de las cuales debe extraerse una muestra representativa, si se quiere garantizar la validez de contenido del instrumento de evaluación. Estos dos elementos proporcionarán unos primeros puntos de referencia a tener en cuenta al diseñar las situaciones de evaluación pertinentes para la evaluación de los conocimientos personales, y también para el diseño de ingenierías adecuadas.

\section{Síntesis y reflexiones finales}

En este artículo hemos presentado una agenda de investigación en educación matemática basada en los principios y herramientas teóricas del EOS. Como se indica en la Sección 3, este sistema teórico asume un paradigma de investigación complejo, basado en una aproximación

\footnotetext{
${ }^{9}$ Empleamos aquí los términos de validez y fiabilidad en su acepción más amplia: ausencia de sesgo y precisión en los procesos de muestreo de situaciones, sujetos, tiempos y circunstancias inevitables en todo proceso de medición educacional y psicológica (FELDT y BRENNAN, 1991; MESSICK, 1991).
} 
holística y sistémica (Cohen et al., 2007), al considerar que es necesario abordar problemas epistemológicos y ontológicos propios de la investigación básica, orientada a la comprensión de los fenómenos, y problemas de diseño instruccional focalizados en la solución de problemas prácticos de la enseñanza y aprendizaje. Abordamos también el problema de la formación de profesores al reconocer el papel fundamental que éstos desempeñan en la implementación de cambios efectivos en la educación matemática mediante actividades de investigación-acción y de práctica reflexiva. Se reconoce, no obstante, la brecha existente entre la investigación científicotecnológica y la práctica reflexiva, la cual puede ser salvada con la Teoría de la Idoneidad Didáctica (TID), el módulo del EOS que aborda la problemática axiológica de identificación y estructuración de los valores y normas de acción para la optimización de los procesos educativos. La TID sienta las bases de un programa de investigación orientado a la identificación de los juicios de valor implicados en cada una de las facetas y componentes de un proceso de instrucción, así como la comparación y articulación de criterios propuestos por distintos marcos teóricos. No se trata de elaborar un único conjunto de criterios generales, sino un sistema de pautas con distintos niveles de generalidad y detalle, según los bloques temáticos de la disciplina correspondiente o incluso a nivel transdisciplinar.

No hemos incluido en este artículo información sobre el origen del EOS, su progresivo desarrollo y las concordancias y complementariedades con otras teorías usadas en educación matemática. Remitimos al lector a las publicaciones realizadas sobre este tema, disponibles en el repositorio web, http://enfoqueontosemiotico.ugr.es/. Sobre el problema metodológico hemos mencionado la necesidad de aplicar diversos métodos cualitativos y cuantitativos según el problema específico investigado. Reconocemos que en el EOS no se ha explorado las posibilidades del software de análisis cualitativo, como, por ejemplo, Atlas.ti, Nudist, Nvivo, para facilitar el análisis de la información que se recoge en los estudios observacionales y en los análisis ontosemióticos de las prácticas matemáticas y didácticas. Igualmente se debiera estudiar con más detalle la aplicación de técnicas de análisis multivariante, como análisis de correspondencias, conglomerados 0 análisis implicativo.

El planteamiento de problemas de investigación en educación matemática es un tema que interesa a los estudiantes de maestría y doctorado, así como a la comunidad de investigación, en particular a los supervisores de tales estudiantes. Además, la formulación de agendas, programas y proyectos de investigación es necesaria para optimizar a medio y largo plazo los esfuerzos de los equipos de investigación. Por otra parte, el planteamiento de problemas significativos requiere, 
además de tener en cuenta el estado del arte en cada caso, usar marcos teóricos y metodológicos que ayuden a hacer operativos y consistentes tales problemas.

El trabajo del profesor de matemáticas en el aula se suele concretar, básicamente, en el diseño, implementación y evaluación de unidades didácticas, por lo que, con frecuencia, los cursos de didáctica de la matemática confluyen en el diseño de tales unidades, ya que permiten motivar y aplicar gran parte de los conocimientos didácticos sobre el tema en el que se centra la unidad. En realidad, el diseño de una unidad didáctica, incluso aunque se haga con fines docentes, es un "trabajo de investigación" mediante el que se sintetizan y aplican conocimientos pedagógicos y conocimientos didácticos. Si el plan de formación contemplara las fases de implementación y evaluación de tales unidades didácticas y se realizara con rigor y sistematicidad se trataría de una investigación didáctica.

El análisis que hemos realizado en este trabajo de las cuestiones docentes referidas a qué matemáticas enseñar y cómo enseñarlas para que los estudiantes aprendan del mejor modo posible, a la luz de los criterios de idoneidad didáctica y del Enfoque Ontosemiótico del conocimiento y la instrucción matemática, sugiere la complejidad de la elaboración de unidades didácticas, y la ingenuidad con la que con frecuencia se abordan.

Los formadores de profesores de matemáticas estamos interesados en sistematizar los conocimientos didácticos disponibles hacia los cuales orientar la preparación de los profesores, así como en la realización y supervisión de investigaciones dirigidas hacia la producción de nuevos conocimientos. Es bien reconocida la complejidad de ambas funciones - docencia e investigación didáctica - ante la variedad de enfoques teóricos y metodológicos disponibles y las diversas disciplinas interesadas en la enseñanza y aprendizaje de las matemáticas. Este trabajo puede ser útil para los investigadores, formadores de profesores y estudiantes de posgrado, los cuales pueden usar los tipos de problemas formulados, en particular, como ayuda en el planteamiento y asignación de trabajos de pre-grado, grado y postgrado.

\section{Reconocimiento}

Trabajo realizado en el marco del Proyecto PID2019-105601GB-I00/AEI/ 10.13039/501100011033 y en el seno del Grupo FQM-126 del PAI (Junta de Andalucía, España).

\section{Referencias:}

ARTEAGA, Pedro; BATANERO, Carmen; GEA, María Magdalena. La componente mediacional del conocimiento didáctico matemático de futuros profesores sobre Estadística: un estudio de evaluación exploratorio. Educação Matemática Debate, v.1, n. 1, p. 54-75, 2017. 
ARTIGUE, Michèle. Perspectives on Design Research: The Case of Didactical Engineering. In $A$. BIKNER-AHSBAHS; C. KNIPPING; N. PRESMEG (Eds), Approaches to Qualitative Research in Mathematics Education. Examples of Methodology and Methods. (pp. 467-496). Springer, 2015.

BAKKER, Arthur; VAN EERDE, Dolly. An introduction to design-based research with an example from statistics education. In Angelika BIKNER-AHSBAHS; Christine KNIPPING; Norma PRESMEG (Eds.), Approaches to qualitative research in mathematics education. Examples of methodology and methods. (pp. 429-466). Springer, 2015.

BATANERO, Carmen; DÍAZ, Carmen. The meaning and understanding of mathematics. The case of probability, In Philosophical dimensions in mathematics education (pp. 107-127). Springer, Boston, M.A., 2007.

BIKNER-AHSBAHS, Angelica; KNIPPING, Christine; PRESMEG, Norma. Approaches to qualitative research in mathematics education. Examples of methodology and methods. Springer, 2015.

RUGINA, Anghel N. The problem of values and value-judgments in science and a positive solution: Max Weber and Ludwig Wittgenstein revisited. International Journal of Social Economics, v. 25, n. 5, p. 805-854, 1998. https://doi.org/10.1108/EUM0000000004522

BREDA, Adriana; FONT, Vicenç; LIMA, Valderez Marina do Rosário. A noção de idoneidade didática e seu uso na formação de professores de matemática. Jornal Internacional de Estudos em Educação Matemática, v. 8, n. 2, p. 1-41, 2015.

BREDA, Adriana; FONT, Vicenç; y PINO-FAN, Luis. Criterios valorativos y normativos en la Didáctica de las Matemáticas: el caso del constructo idoneidad didáctica. Bolema, v. 32, n. 60, p. $255-278,2018$.

BROWN, Ann L. Design experiments: Theoretical and methodological challenges in creating complex interventions in classroom settings. The Journal of the Learning Sciences, v. 2, n. 2, p. 141-178, 1992.

BUNGE, Mario. Las ciencias sociales en discusión: una perspectiva filosófica. Buenos Aires, Editorial Sudamericana, 1999.

BURGOS, María; BELTRÁN-PELLICER, Pablo; GODINO, Juan D. La cuestión de la idoneidad de los vídeos educativos de matemáticas: una experiencia de análisis con futuros maestros de educación primaria. Revista Española de Pedagogía, v. 78, n. 275, p. 27-49, 2020.

BURGOS, María et al. Onto-semiotic complexity of the Definite Integral. Implications for teaching and learning Calculus. REDIMAT - Journal of Research in Mathematics Education, v. 10, n. 1, p. 4-40, 2021.

BURGOS, María y GODINO, Juan D. Modelo ontosemiótico de referencia de la proporcionalidad. Implicaciones para la planificación curricular en primaria y secundaria. AIEM, v. 18, p.1-20, 2020.

CLEMENTS, Ken. Past, present and future dimensions of mathematics education: introduction to the third international handbook of mathematics education. In Ken CLEMENTS; Alan J. BISHOP: Christine KEITEL; Jeremy KILPATRICK; Frederic K. S. LEUNG (Eds.) Third International Handbook of Mathematics Education (pp. v-ix). Springer, 2013. 
COBB, Paul; GRAVEMEIJER, Koeno. Experimenting to support and understand learning processes. In Anthony E. KELLY; Richard A. LESH; John Y. BAEK (Eds.), Handbook of design research methods in education. Innovations in Science, Technology,Engineering and Mathematics Learning and Teaching (pp. 68-95). Mahwah, NJ: Lawrence Erlbaum, 2008.

COHEN, Louis; MANION, Lawrence; MORRISON, Keith. Research methods in education. Routledge, 2007.

D'AMORE, Bruno; FONT, Vicenç; GODINO, Juan D. La dimensión metadidáctica en los procesos de enseñanza y aprendizaje de las matemáticas. Paradigma, v. XXVIII, n. 2, p. 49-77, 2007.

ENGLISH, Lyn D.; KIRSHNER, David. Handbook of International Research in Mathematics Education (3rd edition). Taylor \& Francis, 2016.

FELDT, Leonard S.; BRENNAN, Robert L. Reliability. In R. L. LINN (Ed.), Educational measurement (Third ed.), (pp. 263-331). New York, American Council on Education and Macmillan Publ., 1991.

FONT, Vicenç; CONTRERAS, Ángel. The problem of the particular and its relation to the general in mathematics education. Educational Studies in Mathematics, v. 69, p. 33-52, 2008.

GASCÓN, Josep; NICOLÁS, Pedro. Incidencia de los paradigmas didácticos sobre la investigación didáctica y la práctica docente. Educación Matemática, v. 33, n. 1, p.7-40, 2021.

GEA, María Magdalena; BATANERO, Carmen; ARTEAGA, Pedro; ESTEPA, Antonio. Conocimiento especializado de correlación y regresión en futuros profesores de educación secundaria. Profesorado, Revista de Currículum y Formación del Profesorado, v. 23, p. 397416, 2019.

GIACOMONE, Belén; GODINO, Juan D.; BELTRÁN-PELLICER, Pablo. Desarrollo de la competencia de análisis de la idoneidad didáctica en futuros profesores de matemáticas. Educação e Pesquisa, v. 44, e172011, 2018.

GODINO, Juan D. Un enfoque ontológico y semiótico de la cognición matemática. Recherches en Didactiques des Mathematiques, v. 22, n. (2/3), p. 237-284, 2002.

GODINO, Juan D. Indicadores de la idoneidad didáctica de procesos de enseñanza y aprendizaje de las matemáticas. Cuadernos de Investigación y Formación en Educación Matemática, v. 11, p. 111-132, 2013.

GODINO, Juan D. et al. Didactic engineering as design-based research in mathematics education. In Ubuz, B., Haser, C., \& Mariotti, M. A. (Eds.). Proceedings of the Eighth Congress of the European Society for Research in Mathematics Education (p. 2810-2819) (CERME 8, February 6 - 10, 2013). Ankara, Turkey: Middle East Technical University and ERME, 2013. Disponible en, http://cerme8.metu.edu.tr/wgpapers/WG16/WG16_Godino.pdf

GODINO, Juan D.; BATANERO, Carmen; FONT, Vicenç. The onto-semiotic approach to research in mathematics education. ZDM. The International Journal on Mathematics Education, v. 39, n. 1-2, p. 127-135, 2007. 
GODINO, Juan D.; BATANERO, Carmen; FONT, Vicenç. The onto-semiotic approach: implications for the prescriptive character of didactics. For the Learning of Mathematics, v. 39, n. 1, p. 37- 42, 2019.

GODINO, Juan D.; BURGOS, María. ¿Cómo enseñar las matemáticas y las ciencias experimentales? Resolviendo el dilema de la indagación y transmisión. Paradigma, v. XLI, p. 80 106, 2020.

GODINO, Juan D.; BURGOS, María; GEA, Maria Magdalena. Analysing theories of meaning in mathematics education from the onto-semiotic approach. International Journal of Mathematical Education in Science and Technology, 2021. DOI: 10.1080/0020739X.2021.1896042

GODINO, Juan D.; CONTRERAS, Ángel; FONT, Vicenç. Análisis de procesos de instrucción basado en el enfoque ontológico-semiótico de la cognición matemática. Recherches en Didactiques des Mathematiques, v. 26, n. 1, p. 39-88, 2006.

GODINO, Juan D. et al. Aproximación a la dimensión normativa en Didáctica de la Matemática desde un enfoque ontosemiótico. Enseñanza de las Ciencias, v. 27, n. 1, p. 59-76, 2009.

GODINO, Juan D. et al. Orlando. Why is the learning of elementary arithmetic concepts difficult? Semiotic tools for understanding the nature of mathematical objects. Educational Studies in Mathematics, v. 77, n. 2, p. 247-265, 2011.

GODINO, Juan D. et al. Ingeniería didáctica basada en el enfoque ontológico - semiótico del conocimiento y la instrucción matemáticos, Recherches en Didactique des Mathématiques, $v$. 34, n. 2/3, p. 167-200, 2014.

HART, Lynn C. et al. An examination of research methods in mathematics education. Journal of Mixed Methods Research, v. 3, n. 1, p. 26-41, 2009.

HENRICK, Erin; COBB, Paul; JACKSON, Kara. Educational design research to support systemwide instructional improvement. In Angelica BIKNER-AHSBAHS; Christine KNIPPING; Norma PRESMEG (Eds), Approaches to Qualitative Research in Mathematics Education. Examples of Methodology and Methods. (pp. 497-530). Springer, 2015.

HUMMES, Viviane Beatriz; FONT, Vicenç; BREDA, Adriana. Uso combinado del estudio de clases y la idoneidad didáctica para el desarrollo de la reflexión sobre la propia práctica en la formación de profesores de matemáticas. Acta Scientiae, v. 21, n. 1, p. 64-82, 2019.

INGLIS, Matthew; FOSTER, Colin. Five decades of mathematics education research. Journal for Research in Mathematics Education, v. 49, n. 4, p. 462-500, 2018.

JOHNSON, Burke; ONWUEGBUZIE, Anthony J. Mixed methods research: A research paradigm whose time has come. Educational Researcher, v. 33, n. 7, p. 14-26, 2004.

KELLY, Anthony E.; LESH, Richard A.; BAEK, John Y. (Eds.). Handbook of design research in methods in education. Innovations in science, technology, engineering, and mathematics learning and teaching. New York, NY: Routledge, 2008.

KILPATRICK, Jeremy; SWAFFORD, Jane; FINDELL, Bradford. (Eds.). Adding it up: Helping children learn mathematics. National Academy Press, 2001. 
KORTHAGEN, Fred A. J. The gap between research and practice revisited. Educational Research and Evaluation, v. 13, n. 3, p. $303-310,2007$.

LAMPERT, Magdalene et al. Using Designed Instructional Activities to Enable Novices to Manage Ambitious Mathematics Teaching. In M. K. STEIN; L. KUCAN, (Eds), Instructional Explanations in the Disciplines (pp. 129-141). Springer, 2010.

LERMAN, Stephen. The social turn in mathematics education research. In Joan BOALER (Ed.), Multiple perspectives on mathematics teaching and learning (pp. 19-44). Westport, CT: Ablex, 2000.

MCINTYRE, Donald. Bridging the gap between research and practice. Cambridge Journal of Education, v. 35, n. 3, p. 357-382, 2005.

MESSICK, Samuel Validity. In R. L. LINN (Ed.), Educational measurement (Third ed.), pp. 13104). New York, American Council on Education and Macmillan Publ., 1991.

RAND MATHEMATICS STUDY PANEL. Mathematical proficiency for all students: Toward a strategic research and development program in mathematics education. Santa Monica, CA: Rand, 2003.

SCHOENFELD, Alan H. Methods. In Frank K. Lester (Ed.). Second Handbook of Research on Mathematics Teaching and Learning (pp. 66-110). NCTM \& IAP (Information Age Publishing Inc.), 2007.

SNOW, Richard E.; LOHMAN, David R. Implication of cognitive psychology for educational measurement. In R. L. LINN (Ed.), Educational measurement (Third ed.), pp. 263-331, New York. American Council on Education and Macmillan Publ., 1991.

WEBB, Norman L. Assessment of student's knowledge of mathematics: step toward a theory. In Douglas A. Grouws, (Ed.), Handbook of research on mathematics teaching and learning. New York, Macmillan, 1992.

WEBER, Max. Por qué no se deben hacer juicios de valor en la sociología y en la economía. (Trad. Abellán, J.). Madrid, Alianza Editorial, 1917/2010.

WHEELER, David. Epistemological issues and challenges to assessment: What is mathematical knowledge? In: M. NISS (Ed.), Investigations into assessment in mathematics education. An ICMI Study. Dordrecht, Kluwer A.P., 1993.

WILHELMI, Miguel R.; GODINO, Juan D.; LACASTA, Eduardo. Configuraciones epistémicas asociadas a la noción de igualdad de números reales. Recherches en Didactique des Mathematiques, v. 27, n. 1, p. 77 - 120, 2007.

VYGOTSKY, Lev S. El desarrollo de los procesos psicológicos superiores. Barcelona: CríticaGrijalbo, 1934. 